\title{
REFLEXIONES SOBRE LA EDUCACIÓN A DISTANCIA DESDE LA PERSPECTIVA DEL PROFESOR
}

Comentarios al libro de COPERTARI, Susana. La práctica docente universitaria en Educación a Distancia. Procesos metacognitivos y buena enseñanza. Editorial Laborde. Rosario - Argentina, 2010; 381 páginas.

Sonia B. Concari (Universidad Nacional del Litoral)* sconcari@fiq.unl.edu.ar

Recibido: 29/07/2011 Aceptado: 5/11/2011

La docente e investigadora Susana Copertari (Facultad de Ciencias Políticas y Relaciones Internacionales, y Facultad de Humanidades y Artes de la Universidad Nacional de Rosario) ha publicado recientemente, su libro: "La práctica docente universitaria en Educación a Distancia. Procesos metacognitivos y buena enseñanza", producto de su labor investigativa de los últimos diez años, y de su tesis de Maestría en Docencia Universitaria. El mismo presenta resultados de una experiencia innovadora en Educación a Distancia, describiendo la propuesta de enseñanza y los procesos de reflexión teórica y metodológica sobre su desarrollo en la asignatura Antropología, del primer año de la carrera de Comunicación Social de la Facultad de Ciencias Políticas de la Universidad Nacional de Rosario, durante los años 1999 y 2000.

El libro ofrece una visión retrospectiva de esa experiencia, en la que la autora integra, desde su formación en el campo de las ciencias sociales, y en un proceso de reflexión sobre la praxis, aspectos didácticos, metodológicos y organizativos de un trabajo docente intenso en el que participó activamente. En un texto ameno, cada capítulo muestra un aspecto del proceso de investigación, vinculando el contexto de la experiencia, el objeto de investigación y los agentes involucrados.

* Doctora en Física (UNR). Posgrado en Docencia Universitaria (UNL). Licenciada en Física (UNR). 
El texto se inicia con una introducción en la que se exponen la justificación del estudio, la formulación del problema y los objetivos planteados, a continuación, en el capítulo 1 se describe detalladamente el contexto institucional y académico-curricular correspondientes al momento en el que tuvo lugar la experiencia. El capítulo 2 desarrolla el marco teórico conceptual que sustenta el trabajo, junto con un análisis de las distintas perspectivas con que se aborda la educación a distancia. Se presentan y articulan los aportes de diversos referentes teóricos, para construir el objeto de conocimiento en términos de la integración de las tecnologías de la información y la comunicación a la educación a distancia, la práctica docente, la enseñanza para la comprensión y los procesos metacognitivos.

En el capítulo 3 se presentan los criterios metodológicos adoptados para llevar a cabo el estudio. Se destaca el enfoque interpretativo crítico, planteado desde las perspectivas epistemológica y educativa. Metodológicamente se adoptó el estudio de caso, desarrollado a partir de indagaciones múltiples y complementarias, que incluyeron instrumentos diversos, tales como entrevistas, análisis documental y relatos autobiográficos.

El capítulo 4 da cuenta del intenso trabajo de campo realizado, y de los resultados obtenidos del mismo. La experiencia de la práctica docente en Educación a Distancia, en la cátedra de Antropología, fue realizada desde la perspectiva del pensamiento del profesor, en el marco de la enseñanza para la comprensión. En un relato complejo, pero claro, se expone el proceso recursivo con que fue analizada e interpretada la información obtenida, posibilitando el conocimiento en profundidad de la experiencia.

El último capítulo sintetiza los resultados que la autora construye en ese proceso de articulación permanente entre los conceptos relevantes, los datos y la perspectiva de los propios implicados, valorando la reflexión de los docentes involucrados sobre sus propias prácticas, como producto de la reconstrucción de los hechos del pasado, lo que permitió acreditar las experiencias vividas y los conocimientos construidos.

El libro de Susana Copertari revela un enfoque novedoso en la mirada de las experiencias en educación a distancia, basada en una visión crítica y reflexiva que intenta comprender la experiencia en profundidad. Su trabajo se apoya en un estudio exhaustivo de la experiencia transcurrida durante los años 1999 y 2000, lo que le concede, además, un carácter documental de gran interés para todos los que quieran acceder a una visión de la educación a distancia de esos años.

Como lo señala la autora en la introducción, se trata de una reflexión sobre la enseñanza y los procesos metacognitivos desde el pensamiento del profesor, en el contexto de la educación a distancia.

En síntesis, esta obra constituye un valioso aporte a dos importantes campos de la educación y de la investigación educativa: la educación a distancia y 
la formación docente. La autora ha logrado transmitir un proceso de indagación profunda de caso, desde una perspectiva interpretativa y crítica, empleando técnicas cualitativas y una triangulación intermetodológica exhaustiva, que le han permitido comprender y documentar la práctica docente.

Invito a los futuros profesores, así como a docentes y gestores de programas de educación a distancia, a disfrutar la lectura de este libro. 